\title{
A Least Squares Decomposition Method for Solving Elliptic Equations
}

\author{
By Dennis C. Jespersen
}

\begin{abstract}
This paper analyzes a numerical method for solving second-order elliptic partial differential equations. The idea is to write the equation as a lower-order system and solve the system using least squares techniques. Error estimates are derived for a model problem.
\end{abstract}

1. Introduction. The purpose of this paper is to propose and analyze a numerical method for the solution of second-order elliptic partial differential equations. The method to be presented has its roots in two sources.

The first source is the least squares method for solving elliptic equations due to Bramble and Schatz [3]. This method has the feature that the trial functions are not required to satisfy any boundary conditions. On the other hand, the condition number of the matrix which arises is $O\left(h^{-4 m}\right)$ ffor a $2 m$ th order elliptic problem, the square of the condition number which would arise from usual Galerkin techniques (but this difficulty can be overcome, albeit at the expense of some additional complications [2]). Also, the trial functions are required to be in the Sobolev space $H^{2 m}$ for a $2 m$ th order problem. Thus, the simple piecewise linear trial functions are never admissible.

The second source is the idea of mixed methods for solving partial differential equations, in which the derivatives of the solution are introduced as new independent variables and one attempts to approximate both the solution and its derivatives simultaneously [9]. Mixed methods have the feature that approximations to the derivatives are automatically obtained, and in some problems of physical interest the derivatives are important. Also, less smooth trial functions may be admissible. However, there still may be a problem as to how one treats the boundary conditions. Also, the matrix that arises may be indefinite, and difficult to prove nonsingular.

The method to be studied in this paper is one which combines features of both the above methods. The derivatives of the solution are introduced as new independent variables (the decomposition step), and the resulting system is treated by least squares methods. Thus the smoothness requirement on the trial functions will be relaxed, no boundary conditions will be imposed on the trial functions, and the matrix will be symmetric and positive definite. This idea was apparently first seen in [7] but no analysis of the method was given.

The plan of this paper is as follows. Section 2 contains the necessary technical

Received March 1, 1976; revised December 12, 1976.

AMS (MOS) subject classifications (1970). Primary 65 N30.

Key words and phrases. Rayleigh-Ritz Galerkin methods, least squares approximation, mixed methods.

Copyright $\odot$ 1977, American Mathematical Society 
lemmas. In Section 3 the problem is formulated and in Section 4, the main section, we prove error estimates.

2. Notation and Technical Lemmas. Let $\Omega$ be a bounded region in $R^{N}$ with smooth boundary $\Gamma$ and outward normal $n$. Define the Sobolev spaces $H^{s}(\Omega), H_{0}^{s}(\Omega)$, and $H^{s}(\Gamma)$ for $s \geqslant 0$ as in [6]. Denote the $L^{2}(\Omega)$ inner product of $u$ and $\phi$ by $(u, \phi)$ and the $L^{2}(\Gamma)$ inner product of $\sigma$ and $\tau$ by $\langle\sigma, \tau\rangle$. For $s<0$, define $H^{s}(\Omega)$ as the dual of $H^{-s}(\Omega)$ under the pairing $(u, \phi)$ and $H^{s}(\Gamma)$ as the dual of $H^{-s}(\Gamma)$ under the pairing $\langle\sigma, \tau\rangle$. Let $\|\cdot\|_{s}$ denote the $H^{s}(\Omega)$ norm, $|\cdot|_{s}$ denote the $H^{s}(\Gamma)$ norm. We will also need the space $\mathbf{H}^{s}(\Omega) \equiv H^{s}(\Omega) \times H^{s}(\Omega)$ with inner product $(\mathbf{w}, \mathbf{z})$ and norm $\|\mathbf{w}\|_{s}$. Throughout, $C$ will denote a positive constant not necessarily the same in any two places.

The first two lemmas are from [6] and [1].

LEMmA 2.1. If $\phi \in H^{-s}(\Omega)$ for $s \leqslant 1$, then there is a unique $w \in H^{2-s}(\Omega) \cap$ $H_{0}^{1}(\Omega)$ with $\Delta w=\phi$ in $\Omega$, and $\|w\|_{2-s} \leqslant C\|\phi\|_{-s}$, where $C$ depends only on $\Omega$ and $s$.

Lemma 2.2. (a) If $w \in H^{s}(\Omega)$ for $s>1 / 2$, then $|w|_{s-1 / 2} \leqslant C\|w\|_{s}$, where on the left-hand side we have the trace of $w$ on $\Gamma$, and $C$ depends only on $\Omega$ and s. (b) If $w \in H^{s}(\Omega)$ for $s>3 / 2$, then $|\partial w / \partial n|_{s-3 / 2} \leqslant C\|w\|_{s}$, where $C$ depends only on $\Omega$ and $s$.

Lemma 2.3. There is a constant $C$ such that for all $\epsilon>0$ and for all $w \in H^{1}(\Omega)$, $|w|_{0} \leqslant \epsilon\|w\|_{1}+C\|w\|_{0} / \epsilon$.

Proof. See [4].

Lemma 2.4. For $w \in H^{1}(\Omega)$ and $\mathbf{z} \in \mathbf{H}^{1}(\Omega)$,

$$
(w, \operatorname{div} \mathbf{z})+(\nabla w, \mathbf{z})=\langle w, n \cdot \mathbf{z}\rangle .
$$

Proof. See [10].

Lemma 2.5. If $\tau \in H^{1 / 2}(\Gamma)$, there is a unique $w \in H^{1}(\Omega)$ such that $\Delta w=0$ and $w=\tau$ on $\Gamma$. This $w$ satisfies $\|w\|_{1} \leqslant C|\tau|_{1 / 2}$, where $C$ depends only on $\Omega$.

Proof. See [6].

3. Statement of Problem. Consider the model problem

$$
-\Delta u=f \quad \text { in } \Omega, \quad u=g \text { on } \Gamma,
$$

where $\Gamma, f$, and $g$ are sufficiently smooth. This problem is selected only to keep the formulas which follow as simple as possible; the results hold for more general elliptic problems [5].

Decompose (3.1) into a system of equations

$$
-\nabla u=\mathbf{v} \quad \text { in } \Omega, \quad \operatorname{div} \mathbf{v}=f \quad \text { in } \Omega, \quad u=g \quad \text { on } \Gamma .
$$

Choose, for $0<h \leqslant 1$, finite-dimensional spaces $S_{1}^{h} \subset H^{1}(\Omega), S_{2}^{h} \subset H^{1}(\Omega)$, and define $S_{2}^{h}=S_{2}^{h} \times S_{2}^{h}$. Consider then the problem of minimizing the quadratic functional

$$
J\left(w_{h}, \mathbf{z}_{h}\right) \equiv\left\|\nabla w_{h}+\mathbf{z}_{h}\right\|_{0}^{2}+\left\|\operatorname{div} \mathbf{z}_{h}-f\right\|_{0}^{2}+h^{-1}\left|w_{h}-g\right|_{0}^{2}
$$

$\operatorname{over}\left(w_{h}, \mathbf{z}_{h}\right) \in S_{1}^{h} \times \mathbf{S}_{2}^{h}$.

Define, for $(w, \mathbf{z}),(\phi, \psi) \in H^{1}(\Omega) \times \mathbf{H}^{1}(\Omega)$, a bilinear form by 


$$
A((w, \mathbf{z}),(\phi, \psi))=(\nabla w+\mathbf{z}, \nabla \phi+\psi)+(\operatorname{div} \mathbf{z}, \operatorname{div} \psi)+h^{-1}\langle w, \phi\rangle
$$

where in the $L^{2}(\Gamma)$ inner product we have the restrictions of $w$ and $\phi$ to $\Gamma$. It is classical that $\left(u^{h}, \mathrm{v}^{h}\right)$ minimizes the functional $J$ over $S_{1}^{h} \times \mathrm{S}_{2}^{h}$ if and only if

$$
\begin{aligned}
A\left(\left(u^{h}, \mathbf{v}^{h}\right),\left(\phi_{h}, \psi_{h}\right)\right) & =A\left((u, \mathbf{v}),\left(\phi_{h}, \psi_{h}\right)\right) \\
& =\left(f, \operatorname{div} \psi_{h}\right)+h^{-1}\left\langle g, \phi_{h}\right\rangle
\end{aligned}
$$

for all $\left(\phi_{h}, \psi_{h}\right) \in S_{1}^{h} \times S_{2}^{h}$, where $(u, v)$ is the solution of (3.2). So one finds $u^{h}$ and $\mathbf{v}^{h}$ by solving the finite-dimensional problem (3.4).

The bilinear form $A$ gives rise to a norm by defining $\|(w, \mathbf{z})\|_{A}^{2}=A((w, \mathbf{z}),(w, \mathbf{z}))$. We have the Cauchy-Schwarz inequality for $A,|A((w, \mathbf{z}),(\phi, \psi))| \leqslant\|(w, \mathbf{z})\|_{A}\|(\phi, \psi)\|_{A}$.

Now make the following standard approximability assumptions.

(3.5) There is some $r_{1} \geqslant 2$ such that if $u \in H^{s}(\Omega), 1 \leqslant s \leqslant r_{1}$, then there is some $u_{h} \in S_{1}^{h}$ such that $\left\|u-u_{h}\right\|_{0}+h\left\|u-u_{h}\right\|_{1} \leqslant C h^{s}\|u\|_{s}$ where $C$ is independent of $u$ and $u_{h}$.

(3.6) There is some $r_{2} \geqslant 2$ such that if $\mathbf{v} \in \mathbf{H}^{t}(\Omega), 1 \leqslant t \leqslant r_{2}$, then there is some $\mathbf{v}_{h} \in \mathbf{S}_{2}^{h}$ such that $\left\|\mathbf{v}-\mathbf{v}_{h}\right\|_{0}+h\left\|\mathbf{v}-\mathbf{v}_{h}\right\|_{1} \leqslant C h^{t}\|\mathbf{v}\|_{t}$ where $C$ is independent of $\mathbf{v}$ and $\mathbf{v}_{h}$.

These assumptions are satisfied by the finite-dimensional spaces used in practice.

Lemma 3.1. Let $(u, \mathbf{v})$ be the solution of (3.2), where $f \in H^{k}(\Omega)$ and $g \in$ $H^{k+3 / 2}(\Gamma), k \geqslant 0$. Then a unique solution $\left(u^{h}, v^{h}\right)$ of $(3.4)$ exists, and

$$
\begin{aligned}
& \left\|\left(u-u^{h}, \mathbf{v}-\mathbf{v}^{h}\right)\right\|_{A}=\inf _{\left(w^{h}, z^{h}\right) \in S_{1}^{h} \times \mathrm{s}_{2}^{h}}\left\|\left(u-w^{h}, \mathbf{v}-\mathbf{z}^{h}\right)\right\|_{A} \\
& \leqslant C\left(h^{s-1}\|u\|_{s}+h^{t-1}\|\mathbf{v}\|_{t}\right), \quad s \leqslant \min \left(k+2, r_{1}\right), t \leqslant \min \left(k+1, r_{2}\right) .
\end{aligned}
$$

Proof. From (3.4) we have the fundamental orthogonality relation

$$
A\left(\left(u-u^{h}, \mathbf{v}-\mathbf{v}^{h}\right),\left(\phi_{h}, \psi_{h}\right)\right)=0 \quad \text { for all }\left(\phi_{h}, \psi_{h}\right) \in S_{1}^{h} \times \mathbf{S}_{2}^{h} .
$$

Thus, $\left(u^{h}, \mathbf{v}^{h}\right)$ is the projection of $(u, \mathbf{v})$ onto $S_{1}^{h} \times S_{2}^{h}$ in the $A$-inner product, and so we have existence, uniqueness (since the $A$-inner product is nondegenerate) and the first part of (3.7). For the second part of (3.7), note that

$$
\begin{aligned}
&\left\|\left(u-w^{h}, \mathbf{v}-\mathbf{z}^{h}\right)\right\|_{A} \leqslant\left\|\nabla\left(u-w^{h}\right)+\left(\mathbf{v}-\mathbf{z}^{h}\right)\right\|_{0}+\left\|\operatorname{div}\left(\mathbf{v}-\mathbf{z}^{h}\right)\right\|_{0}+h^{-1 / 2}\left|u-w^{h}\right|_{0} \\
& \leqslant\left\|u-w^{h}\right\|_{1}+\left\|\mathbf{v}-\mathbf{z}^{h}\right\|_{0}+C\left\|\mathbf{v}-\mathbf{z}^{h}\right\|_{1} \\
&+h^{-1 / 2}\left(C h^{-1 / 2}\left\|u-w^{h}\right\|_{0}+h^{1 / 2}\left\|u-w^{h}\right\|_{1}\right) \\
& \quad \text { by Lemma } 2.3 \text { with } \epsilon=h^{1 / 2} \\
& \leqslant
\end{aligned}
$$

Taking the infimum over all $\left(w^{h}, \mathbf{z}^{h}\right) \in S_{1}^{h} \times \mathbf{S}_{2}^{h}$, we obtain the second half of (3.7), and the proof is complete.

Note that if we take $r_{1}=r_{2}=r$ (i.e., use piecewise polynomials of the same degree $r-1$ to approximate $u$ and $\mathbf{v}$ ), then 


$$
\left\|\left(u-u^{h}, \mathbf{v}-\mathbf{v}^{h}\right)\right\|_{A} \leqslant C h^{r-1}\left(\|u\|_{r}+\|\mathbf{v}\|_{r}\right) \leqslant C h^{r-1}\left(\|f\|_{r-1}+|g|_{r+1 / 2}\right) .
$$

4. Error Estimates. In this section we derive estimates for the errors $e_{u} \equiv$ $u-u^{h}$ and $\mathrm{e}_{v} \equiv \mathbf{v}-\mathbf{v}^{h}$. The key tools are the orthogonality relation (3.8) and the inequality

$$
\begin{aligned}
& \|\nabla w+\mathbf{z}\|_{0}+\|\operatorname{div} \mathbf{z}\|_{0}+h^{-1 / 2}|w|_{0} \\
& \leqslant C\|(w, z)\|_{A} \leqslant C\left(\|w\|_{1}+h^{-1}\|w\|_{0}+\|z\|_{0}+\|\operatorname{div} z\|_{0}\right),
\end{aligned}
$$

which is an easy consequence of the definition of $\|(w, z)\|_{A}$.

Lemma 4.1. For all $\phi \in H^{1}(\Omega),\left|\left(\operatorname{div} \mathrm{e}_{v}, \phi\right)\right| \leqslant C h\left\|\left(e_{u}, \mathrm{e}_{v}\right)\right\|_{A}\|\phi\|_{1}$.

Proof. Given $\phi \in H^{1}(\Omega)$, let $\alpha$ solve $-\Delta \alpha=\phi$ in $\Omega, \alpha=0$ on $\Gamma$, and let $\beta=$ $-\nabla \alpha$, so $\operatorname{div} \beta=\phi$. Then $\|\alpha\|_{3} \leqslant C\|\phi\|_{1}$. Now we have

$$
\left(\operatorname{div} \mathbf{e}_{v}, \phi\right)=\left(\operatorname{div} \mathbf{e}_{v}, \operatorname{div} \beta\right)=A\left(\left(e_{u}, e_{v}\right),(\alpha, \beta)\right)=A\left(\left(e_{u}, \mathbf{e}_{v}\right),\left(\alpha-\alpha_{h}, \beta-\beta_{h}\right)\right)
$$

for all $\left(\alpha_{h}, \beta_{h}\right) \in S_{1}^{h} \times S_{2}^{h}$. Thus,

$$
\begin{aligned}
\left|\left(\operatorname{div} \mathrm{e}_{v}, \phi\right)\right| & \leqslant\left\|\left(e_{u}, \mathrm{e}_{v}\right)\right\|_{A}\left\|\left(\alpha-\alpha_{h}, \beta-\beta_{h}\right)\right\|_{A} \\
& \leqslant\left\|\left(e_{u}, \mathrm{e}_{v}\right)\right\|_{A} C\left(h\|\alpha\|_{2}+h\|\beta\|_{2}\right) \leqslant C h\left\|\left(e_{u}, \mathrm{e}_{v}\right)\right\|_{A}\|\alpha\|_{3} \\
& \leqslant C h\left\|\left(e_{u}, \mathbf{e}_{v}\right)\right\|_{A}\|\phi\|_{1} .
\end{aligned}
$$

Note that this shows div $\mathrm{e}_{v}$, considered as an element of $H^{-1}(\Omega)$, has norm bounded by $C h\left\|\left(e_{u}, \mathbf{e}_{v}\right)\right\|_{A}$.

LEMmA 4.2. $\left|e_{u}\right|_{-1 / 2} \leqslant C h\left\|\left(e_{u}, \mathbf{e}_{v}\right)\right\|_{A}$.

Proof. By definition

$$
\left|e_{u}\right|_{-1 / 2}=\sup _{\tau \in H^{1 / 2}(\Gamma)} \frac{\left\langle e_{u}, \tau\right\rangle}{|\tau|_{1 / 2}} .
$$

Given $\tau \in H^{1 / 2}(\Gamma)$, let $\alpha \in H^{1}(\Omega)$ solve $\Delta \alpha=0$ in $\Omega, \alpha=\tau$ on $\Gamma$. This can be done by Lemma 2.5 , and $\|\alpha\|_{1} \leqslant C|\tau|_{1 / 2}$. Now, for all $\alpha_{h} \in S_{1}^{h}$,

$$
\begin{aligned}
\left\langle e_{u}, \tau\right\rangle & =\left\langle e_{u}, \alpha\right\rangle=h\left(h^{-1}\left\langle e_{u}, \alpha\right\rangle\right)=h\left(h^{-1}\left\langle e_{u}, \alpha\right\rangle-A\left(\left(e_{u}, \mathbf{e}_{v}\right),\left(\alpha_{h}, 0\right)\right)\right) \\
& =h\left(h^{-1}\left\langle e_{u}, \alpha-\alpha_{h}\right\rangle-\left(\nabla e_{u}+\mathrm{e}_{v}, \nabla \alpha_{h}\right)\right) .
\end{aligned}
$$

Thus we have

$$
\begin{aligned}
\left|\left\langle e_{u}, \tau\right\rangle\right| & \leqslant h\left(h^{-1 / 2}\left|e_{u}\right|_{0} h^{-1 / 2}\left|\alpha-\alpha_{h}\right|_{0}+\left\|\nabla e_{u}+\mathbf{e}_{v}\right\|_{0}\left\|\nabla \alpha_{h}\right\|_{0}\right) \\
& \leqslant C h\left\|\left(e_{u}, \mathbf{e}_{v}\right)\right\|_{A}\left(h^{-1}\left\|\alpha-\alpha_{h}\right\|_{0}+\left\|\alpha-\alpha_{h}\right\|_{1}+\left\|\alpha_{h}\right\|_{1}\right) \\
& \leqslant C h\left\|\left(e_{u}, \mathbf{e}_{v}\right)\right\|_{A}\left(h^{-1}\left\|\alpha-\alpha_{h}\right\|_{0}+\left\|\alpha-\alpha_{h}\right\|_{1}+\|\alpha\|_{1}\right),
\end{aligned}
$$

where we again used Lemma 2.3. Now taking the infimum over $\alpha_{h} \in S_{1}^{h}(\Omega)$ bounds this by $C h\left\|\left(e_{u}, \mathbf{e}_{v}\right)\right\|_{A}\|\alpha\|_{1} \leqslant C h\left\|\left(e_{u}, \mathbf{e}_{v}\right)\right\|_{A}|\tau|_{1 / 2}$, which finishes the proof.

Corollary. For $0 \leqslant s \leqslant 1 / 2,\left|e_{u}\right|_{s-1 / 2} \leqslant C h^{1-s}\left\|\left(e_{u}, \mathbf{e}_{v}\right)\right\|_{A}$.

Proof. We have $\left|e_{u}\right|_{0} \leqslant C h^{1 / 2}\left\|\left(e_{u}, \mathbf{e}_{v}\right)\right\|_{A}$ and $\left|e_{u}\right|_{-1 / 2} \leqslant C h\left\|\left(e_{u}, \mathbf{e}_{v}\right)\right\|_{A}$.

The corollary follows by interpolation. 
TheOREM 4.1. For $0 \leqslant s<1 / 2,\left\|u-u^{h}\right\|_{s} \leqslant C h^{1-s}\left\|\left(e_{u}, \mathbf{e}_{v}\right)\right\|_{A}$.

Proof. The proof uses the duality argument of Nitsche [8]. We have

$$
\left\|e_{u}\right\|_{s}=\sup _{\phi \in H^{-s}(\Omega)} \frac{\left(e_{u}, \phi\right)}{\|\phi\|_{-s}}
$$

Given $\phi \in H^{-s}(\Omega)$, let $w \in H^{2-s}(\Omega)$ solve $-\Delta w=\phi$ in $\Omega, w=0$ on $\Gamma$. By Lemma $2.1,\|w\|_{2-s} \leqslant C\|\phi\|_{-s}$. We have

$$
\begin{aligned}
\left(e_{u}, \phi\right) & =\left(e_{u},-\Delta w\right)=\left(\nabla e_{u}, \nabla w\right)-\left\langle e_{u}, \frac{\partial w}{\partial n}\right\rangle \\
& =\left(\nabla e_{u}+\mathbf{e}_{v}, \nabla w\right)-\left(\mathbf{e}_{v}, \nabla w\right)-\left\langle e_{u}, \frac{\partial w}{\partial n}\right\rangle \\
& =A\left(\left(e_{u}, \mathbf{e}_{v}\right),(w, 0)\right)+\left(\operatorname{div} \mathrm{e}_{v}, w\right)-\left\langle e_{u}, \frac{\partial w}{\partial n}\right\rangle \\
& =A\left(\left(e_{u}, \mathbf{e}_{v}\right),\left(w-w_{h}, 0\right)\right)+\left(\operatorname{div} \mathrm{e}_{v}, w\right)-\left\langle e_{u}, \frac{\partial w}{\partial n}\right\rangle
\end{aligned}
$$

for all $w_{h} \in S_{1}^{h}(\Omega)$. Now estimating each term on the right-hand side separately, we have with the help of (4.1), Lemma 4.1 and the corollary to Lemma 4.2 ,

$$
\begin{aligned}
\left|\left(e_{u}, \phi\right)\right| \leqslant & \left\|\left(e_{u}, \mathrm{e}_{v}\right)\right\|_{A}\left\|\left(w-w_{h}, 0\right)\right\|_{A}+\left|\left(\operatorname{div} \mathrm{e}_{v}, w\right)\right|+\left|e_{u}\right|_{s-1 / 2}\left|\frac{\partial w}{\partial n}\right|_{1 / 2-s} \\
\leqslant & \left\|\left(e_{u}, \mathrm{e}_{v}\right)\right\|_{A} C\left(\left\|w-w_{h}\right\|_{1}+h^{-1}\left\|w-w_{h}\right\|_{0}\right) \\
& +C h\left\|\left(e_{u}, \mathrm{e}_{v}\right)\right\|_{A}\|w\|_{1}+C h^{1-s}\left\|\left(e_{u}, \mathrm{e}_{v}\right)\right\|_{A}\|w\|_{2-s} .
\end{aligned}
$$

Taking the infimum over $w_{h} \in S_{1}^{h}$ yields

$$
\begin{aligned}
\left|\left(e_{u}, \phi\right)\right| \leqslant & \left\|\left(e_{u}, \mathbf{e}_{v}\right)\right\|_{A} C h^{1-s}\|w\|_{2-s}+C h\left\|\left(e_{u}, \mathbf{e}_{v}\right)\right\|_{A}\|w\|_{1} \\
& +C h^{1-s}\left\|\left(e_{u}, \mathbf{e}_{v}\right)\right\|_{A}\|w\|_{2-s} \\
\leqslant & C h^{1-s}\left\|\left(e_{u}, \mathbf{e}_{v}\right)\right\|_{A}\|w\|_{2-s} \leqslant C h^{1-s}\left\|\left(e_{u}, \mathbf{e}_{v}\right)\right\|_{A}\|\phi\|_{-s},
\end{aligned}
$$

which completes the proof.

Theorem 4.1 implies that $\left\|u-u^{h}\right\|_{0}$ is of optimal order in $h$ provided $f$ and $g$ are smooth enough and $r_{2} \geqslant r_{1}$. We now come to the question of an error estimate for $\mathrm{e}_{v}$. The following a priori estimate is our starting point.

LEMmA 4.3. For all $(w, \mathbf{z}) \in H^{1}(\Omega) \times \mathbf{H}^{1}(\Omega)$,

$$
\|w\|_{1}+\|\mathrm{z}\|_{0} \leqslant C\left(\|\nabla w+\mathrm{z}\|_{0}+\|\operatorname{div} \mathrm{z}\|_{-1}+|w|_{1 / 2}\right) .
$$

Proof. Given $\phi \in H^{-1}(\Omega)$, let $\alpha \in H_{0}^{1}(\Omega)$ solve $-\Delta \alpha=\phi$ in $\Omega$. It follows from results in [1] that we may define $\partial \alpha / \partial n$ as an element of $H^{-1 / 2}(\Gamma)$, and $|\partial \alpha / \partial n|_{-1 / 2}$ $\leqslant C\|\alpha\|_{1} \leqslant C\|\phi\|_{-1}$. Then the same computation as in Theorem 4.1 gives

$$
(w, \phi)=(\nabla w+\mathbf{z}, \nabla \alpha)+(\operatorname{div} \mathbf{z}, \alpha)-\left\langle w, \frac{\partial \alpha}{\partial n}\right\rangle .
$$


Taking absolute values gives the bound for $\|w\|_{1}$; for $\mathbf{z}$, simply write $\mathbf{z}=(\nabla w+\mathbf{z})-$ $\nabla w$ and take norms.

To take advantage of the estimate of Lemma 4.3, we introduce another assumption.

(4.2) Assume there exists a $C>0$ such that $\left|w_{h}\right|_{1 / 2} \leqslant C h^{-1 / 2}\left|w_{h}\right|_{0}$ as $h \rightarrow 0$ for all $w_{h} \in S_{1}^{h}$. The assumption (4.2) is an assumption of inverse type [1] on the elements of $S_{1}^{h}$ restricted to the boundary.

THEOREM 4.2. Suppose the boundary inverse hypothesis (4.2) holds. Then

$$
\left\|e_{u}\right\|_{1}+\left\|\mathbf{e}_{v}\right\|_{0} \leqslant C\left(\left\|\left(e_{u}, \mathbf{e}_{v}\right)\right\|_{A}+\inf _{\phi_{h} \in S_{1}^{h}}\left(h^{-1}\left\|u-\phi_{h}\right\|_{0}+\left\|u-\phi_{h}\right\|_{1}\right)\right) .
$$

Proof. We begin by bounding $\left|e_{u}\right|_{1 / 2}$. Indeed, for all $\phi_{h} \in S_{1}^{h}$,

$$
\begin{aligned}
\left|u-u^{h}\right|_{1 / 2} & \leqslant\left|u-\phi_{h}\right|_{1 / 2}+\left|\phi_{h}-u^{h}\right|_{1 / 2} \leqslant\left|u-\phi_{h}\right|_{1 / 2}+C h^{-1 / 2}\left|\phi_{h}-u^{h}\right|_{0} \\
& \leqslant C\left\|u-\phi_{h}\right\|_{1}+C h^{-1 / 2}\left(\left|\phi_{h}-u\right|_{0}+\left|u-u^{h}\right|_{0}\right) \\
& \leqslant C\left\|u-\phi_{h}\right\|_{1}+C h^{-1}\left\|\phi_{h}-u\right\|_{0}+C\left\|\phi_{h}-u\right\|_{1}+C\left\|\left(e_{u}, \mathbf{e}_{v}\right)\right\|_{A},
\end{aligned}
$$

so we have

$$
\left|e_{u}\right|_{1 / 2} \leqslant C\left(\left\|\left(e_{u}, \mathbf{e}_{v}\right)\right\|_{A}+\inf _{\phi_{h} \in S_{1}^{h}}\left(h^{-1}\left\|u-\phi_{h}\right\|_{0}+\left\|u-\phi_{h}\right\|_{1}\right)\right) .
$$

Now Lemma 4.3, Eq. (4.3), and the observation that $\|\phi\|_{-1} \leqslant\|\phi\|_{0}$ for all $\phi \in H^{0}(\Omega)$ give us

$$
\begin{aligned}
\left\|e_{u}\right\|_{1}+\left\|\mathrm{e}_{v}\right\|_{0} & \leqslant C\left(\left\|\nabla e_{u}+\mathbf{e}_{v}\right\|_{0}+\left\|\operatorname{div} \mathbf{e}_{v}\right\|_{0}+\left|e_{u}\right|_{1 / 2}\right) \\
& \leqslant C\left(\left\|\left(e_{u}, \mathbf{e}_{v}\right)\right\|_{A}+\inf _{\phi_{h} \in S_{1}^{h}}\left(h^{-1}\left\|u-\phi_{h}\right\|_{0}+\left\|u-\phi_{h}\right\|_{1}\right)\right),
\end{aligned}
$$

finishing the proof.

This is an estimate of optimal order for $\left\|e_{u}\right\|_{1}$ but of suboptimal order for $\left\|e_{v}\right\|_{0}$. It is unclear if this suboptimal bound is sharp.

Finally, let us consider the condition number of the matrix problem (3.4). The entries of the matrix, call it $A$, are various inner products of basis elements of the spaces $S_{1}^{h}$ and $\mathbf{S}_{2}^{h}$. Suppose $\left\{w_{1}, \ldots, w_{n}\right\}$ is a basis for $S_{1}^{h}$ and $\left\{\mathbf{z}_{1}, \ldots, \mathbf{z}_{m}\right\}$ is a basis for $S_{2}^{h}$. Then $A$ is a symmetric positive definite $(m+n) \times(m+n)$ matrix whose entries $a_{i j}$ are given by

$$
\begin{aligned}
& a_{i j}=A\left(\left(w_{i}, 0\right),\left(w_{j}, 0\right)\right)=\left(\nabla w_{i}, \nabla w_{j}\right)+h^{-1}\left\langle w_{i}, w_{j}\right\rangle \text { for } 1 \leqslant i, j \leqslant n, \\
& a_{i j}=A\left(\left(w_{i}, 0\right),\left(0, \mathbf{z}_{j-n}\right)\right)=\left(\nabla w_{i}, \mathbf{z}_{j-n}\right)=a_{j i} \text { for } 1 \leqslant i \leqslant n, n+1 \leqslant j \leqslant m+n, \\
& a_{i j}=A\left(\left(0, \mathbf{z}_{i-n}\right),\left(0, \mathbf{z}_{j-n}\right)\right)=\left(\mathbf{z}_{i-n}, \mathbf{z}_{j-n}\right)+\left(\operatorname{div} \mathbf{z}_{i-n}, \operatorname{div} \mathbf{z}_{j-n}\right) \\
& \quad \text { for } n+1 \leqslant i, j \leqslant m+n .
\end{aligned}
$$

We now assume the bases are chosen so that the following conditions hold [2] . Recall $\Omega \subset R^{N}$; the notation $(\cdot, \cdot)_{1}$ denotes the $H^{1}(\Omega)$ inner product.

For all real numbers $\xi_{1}, \ldots, \xi_{n}, \eta_{1}, \ldots, \eta_{m}$, 

(a) $c h^{N} \sum \xi_{i}^{2} \leqslant \sum\left(w_{i}, w_{j}\right) \xi_{i} \xi_{j} \leqslant C h^{N} \sum \xi_{i}^{2}$,
(b) $c h^{N} \sum \eta_{i}^{2} \leqslant \sum\left(\mathbf{z}_{i}, \mathbf{z}_{j}\right) \eta_{i} \eta_{j} \leqslant C h^{N} \sum \eta_{i}^{2}$,
(c) $\sum\left(w_{i}, w_{j}\right)_{1} \xi_{i} \xi_{j} \leqslant C h^{N-2} \sum \xi_{i}^{2}$,
(d) $\sum\left(\mathrm{z}_{i}, \mathbf{z}_{j}\right)_{1} \eta_{i} \eta_{j} \leqslant C h^{N-2} \sum \eta_{i}^{2}$.

Now we have

THEOREM 4.3. Suppose the boundary inverse hypothesis (4.2) and the basis conditions (4.4) hold. Then the condition number of the matrix $A$ is $O\left(h^{-2}\right)$, i.e., cond $(A) \leqslant C h^{-2}$.

Proof. Since $A$ is symmetric positive definite, cond $(A)=\lambda_{\max }(A) / \lambda_{\min }(A)$, the maximum eigenvalue of $A$ divided by the minimum eigenvalue. We estimate the eigenvalues by considering the Rayleigh quotient $\xi^{T} A \xi / \xi^{T} \xi$. Let $\xi=\left(\xi_{1}, \ldots, \xi_{n}\right.$, $\left.\eta_{1}, \ldots, \eta_{m}\right)$ be an $(m+n)$-component vector. Define $w_{h}=\Sigma \xi_{i} w_{i}, \mathbf{z}_{h}=\Sigma \eta_{i} \mathbf{z}_{i}$. Then $\xi^{T} A \xi=A\left(\left(w_{h}, \mathbf{z}_{h}\right),\left(w_{h}, \mathbf{z}_{h}\right)\right)$, and we have

$$
\begin{aligned}
& C \cdot A\left(\left(w_{h}, \mathbf{z}_{h}\right),\left(w_{h}, \mathbf{z}_{h}\right)\right) \geqslant\left\|w_{h}\right\|_{1}^{2}+\left\|\mathbf{z}_{h}\right\|_{0}^{2} \geqslant\left\|w_{h}\right\|_{0}^{2}+\left\|\mathbf{z}_{h}\right\|_{0}^{2} \\
&=\sum \xi_{i} \xi_{j}\left(w_{i}, w_{j}\right)+\sum \eta_{i} \eta_{j}\left(\mathbf{z}_{i}, \mathbf{z}_{j}\right) \geqslant c h^{N}\left(\sum \xi_{i}^{2}+\sum \eta_{i}^{2}\right)=c h^{N} \xi^{T} \xi .
\end{aligned}
$$

The first step here used Lemma 4.3 and the assumption (4.2). We also have

$$
\begin{aligned}
A\left(\left(w_{h}, \mathbf{z}_{h}\right),\right. & \left.\left(w_{h}, \mathbf{z}_{h}\right)\right) \leqslant C\left(h^{-2}\left\|w_{h}\right\|_{0}^{2}+\left\|w_{h}\right\|_{1}^{2}+\left\|\mathbf{z}_{h}\right\|_{0}^{2}+\left\|\mathbf{z}_{h}\right\|_{1}^{2}\right) \\
& \leqslant C\left(h^{-2}\left\|w_{h}\right\|_{0}^{2}+\left\|w_{h}\right\|_{1}^{2}+\left\|\mathbf{z}_{h}\right\|_{1}^{2}\right) \\
& \leqslant C\left(h^{-2} \sum \xi_{i} \xi_{j}\left(w_{i}, w_{j}\right)+\sum \xi_{i} \xi_{j}\left(w_{i}, w_{j}\right)_{1}+\sum \eta_{i} \eta_{j}\left(\mathbf{z}_{i}, \mathbf{z}_{j}\right)_{1}\right) \\
& \leqslant C\left(h^{N-2} \sum \xi_{i}^{2}+h^{N-2} \sum \eta_{i}^{2}\right) \leqslant C h^{N-2} \xi^{T} \xi .
\end{aligned}
$$

Hence $\lambda_{\max }(A) \leqslant C h^{N-2}$ and $\lambda_{\min }(A) \geqslant c h^{N}$, and the proof is complete.

In conclusion, it is clear that the least squares decomposition idea of this paper can be applied to virtually any partial differential equation or system of equations. The analysis of cases other than the elliptic remains a project for the future.

Acknowledgement. I would like to thank my thesis advisor, George Fix, for his constant encouragement.

\footnotetext{
Department of Mathematics

Oregon State University

Corvallis, Oregon 97331
}

1. IVO BABUŠKA \& A. K. AZIZ, "Survey lectures on the mathematical foundations of the finite element method," The Mathematical Foundations of the Finite Element Method with Applications to Partial Differential Equations (A. K. Aziz, Editor), Academic Press, New York, 1972, pp. 3-359. MR 49 \#11824.

2. J. H. BRAMBLE \& J. A. NITSCHE, “A generalized Ritz-least-squares method for Dirichlet problems," SIAM J. Numer. Anal., v. 10, 1973, pp. 81-93. MR 47 \#2836.

3. J. H. BRAMBLE \& A. H. SCHATZ, "Least squares methods for $2 m$ th order elliptic boundary-value problems," Math. Comp., v. 25, 1971, pp. 1-32. MR 45 \#4657.

4. J. BRAMBLE \& V. THOMÉE, "Semidiscrete least-squares methods for a parabolic boundary value problem," Math. Comp., v. 26, 1972, pp. 633-648. MR 50 \# 1532. 
5. D. JESPERSEN, A Least Squares Decomposition Method for the Numerical Solution of Elliptic Partial Differential Equations, Dissertation, University of Michigan, 1976.

6. J.-L. LIONS \& E. MAGENES, Problèmes aux Limites Non Homogènes et Applications. Vol. 1, Dunod, Paris, 1968. MR 40 \# 512.

7. P. P. LYNN \& S. K. ARYA, "Use of the least squares criterion in the finite element formulation," Internat. J. Numer. Methods Engrg., v. 6, 1973, pp. 75-88. MR 48 \# 7752.

8. J. A. NITSCHE, "Ein Kriterium für die Quasi-Optimalität des Ritzschen Verfahrens," Numer. Math., v. 11, 1968, pp. 346-348. MR 38 \# 1823.

9. J. N. REDDY \& J. T. ODEN, "Mixed finite-element approximations of linear boundaryvalue problems," Quart. Appl. Math., v. 33, 1975, pp. 255-280.

10. R. TEMAM, On the Theory and Numerical Analysis of the Navier-Stokes Equations, Lecture Notes, University of Maryland, 1973. 